\title{
Comparison of US Pharmacopeia Simulated Intestinal Fluid TS (without pancreatin) and Phosphate Standard Buffer pH 6.8, TS of the International Pharmacopoeia with Respect to Their Use in In Vitro Dissolution Testing

Erika Stippler ${ }^{1,2}$, Sabine Kopp ${ }^{3,4}$, Jennifer B. Dressman ${ }^{1,5}$

Introduction

uffer solutions suitable for dissolution tests are described as such only in The United States Pharmacopeia (USP); The International Pharmacopoeia (IntPh), for example, broadly describes the composition of buffer solutions without specifying their fields of applicability. In this study Simulated Intestinal Fluid (SIFsp), which is described in The 26th United States Pharmacopeia (USP 26) as an 0.05 M buffer solution containing potassium dihydrogen phosphate (the pancreatin is omitted from the composition for most dissolution testing applications), and Phosphate Standard Buffer pH 6.8TS, which is described in Volume 1 of The International Pharmacopoeia,Third Edition, (IntPh 3) as a mixture of $0.025 \mathrm{M}$ potassium dihydrogen phosphate buffer solution and $0.025 \mathrm{M}$ di-sodium hydrogen phosphate buffer solution, were investigated with respect to their interchangeability as dissolution test media.

Both are $\mathrm{pH} 6.8$ phosphate buffers, and they have similar osmolalities (114 mOsmol $/ \mathrm{kg})$ and buffer capacities (18 mEq/L/pH/unit). However, in the USP medium potassium $(50 \mathrm{mM})$ dominates over sodium $(22 \mathrm{mM})$ whereas in the IntPh buffer sodium $(49.7 \mathrm{mM})$ dominates over potassium $(25 \mathrm{mM})$. [Note: For the current investigations the IntPh phosphate buffer media was used as monographed in the 3rd edition, Volume 1. With the publication of Volume 5 the composition of the buffer $\mathrm{pH} 6.8$ medium has been changed. Volume 5 describes a $0.04 \mathrm{M}$ disodium hydrogen phosphate buffer solution for Buffer pH 6.8.].

The applicability and the interchangeability of these two compositionally similar but not identical buffers as dissolution media was investigated for products containing one of three drug substances: Metronidazole, a typical BCS Class 1 drug, and indometacin and ibuprofen, two drugs belonging to

'J. W. Goethe University Frankfurt/Main, Department of

Pharmaceutical Technology, Germany

2 PHAST GmbH, Homburg/Saar, Germany

${ }^{3}$ World Health Organization, Geneva, Switzerland

${ }^{4}$ The opinions expressed in this article are those of the author and
BCS Class 2. In each case several commercial products currently available on the German market were investigated. Products included uncoated, film coated, and sugar coated tablets as well as hard gelatin capsules.

\section{Experimental Investigations}

Materials

Chemical reference standard substances (CRS) Metronidazole CRS (lot 00616) was purchased from Aldrich (Germany), indometacin CRS (lot 0111A079) was purchased from Synopharm (Germany), and ibuprofen CRS (lot 10337012) was purchased from Caelo (Germany).

Reagents

All reagents used were of analytical grade and were purchased from VWR (Germany).

\section{Dissolution media}

The compositions of dissolution media used for in vitro dissolution testing are described in Table 1. The reagents were added to the deionized water under stirring to yield clear solutions. The $\mathrm{pH}$ value of the dissolution media was checked and adjusted as necessary to $\mathrm{pH} 6.8 \pm 0.05$ with $0.1 \mathrm{M}$ sodium hydroxide or $0.1 \mathrm{M}$ phosphoric acid solution.

Pharmaceutical preparations

The pharmaceutical preparations (all conventional immediate release solid oral dosage forms) investigated are described in Table 2.

Analytical instruments

Dissolution tests were performed with the USP 26 apparatus 2, using an Erweka model DT6R (Erweka, Germany) apparatus. For manual sampling $5 \mathrm{~mL}$ syringes fitted with stainless tubing were used. Samples were filtered immediately after sampling through PTFE filter sticks with $45 \mu \mathrm{m}$ do not necessarily represent the decisions or stated policy of the World Health Organization.

${ }^{5}$ Correspondending author, J. W. Goethe University Frankfurt/Main, Department of Pharmaceutical Technology, Biozentrum, MarieCurie-Str. 9, 60439 Frankfurt, Germany 
Table 1: Composition of dissolution media for in vitro dissolution testing

\begin{tabular}{|c|c|c|}
\hline Medium & Composition & \\
\hline $\begin{array}{l}\text { Simulated Intestinal } \\
\text { Fluid, pH } 6.8^{1} \text { (SIFsp); } \\
\text { USP } 26\end{array}$ & $\begin{array}{l}\mathrm{KH}_{2} \mathrm{PO}_{4} \\
\mathrm{NaOH} \\
\text { deionized water to }\end{array}$ & $\begin{array}{l}68.05 \mathrm{~g} \\
8.96 \mathrm{~g} \\
10.0 \mathrm{~L}\end{array}$ \\
\hline $\begin{array}{l}\text { Phosphate Standard } \\
\text { Buffer pH } 6.8^{2} \\
\text { IntPh } 3\end{array}$ & $\begin{array}{l}\mathrm{KH}_{2} \mathrm{PO}_{4} \\
\mathrm{Na}_{2} \mathrm{HPO}_{4} \\
\text { deionized water to }\end{array}$ & $\begin{array}{l}34.0 \mathrm{~g} \\
35.3 \mathrm{~g} \\
10.0 \mathrm{~L}\end{array}$ \\
\hline \multicolumn{3}{|c|}{$\begin{array}{l}{ }^{1} \text { The composition of SIFsp is the same as in USP } 25 . \\
{ }^{2} \text { The composition of the Phosphate Standard Buffer } \mathrm{pH} 6.8 \text { has been } \\
\text { changed with the publication of Volume } 5 \text { of the IntPh. }\end{array}$} \\
\hline
\end{tabular}

For measurements of osmotic pressure a semi-micro osmometer (Knauer, Germany) was used. The semi-micro osmometer was calibrated before use over the range from $0 \mathrm{mOsmol} / \mathrm{kg}$ to $400 \mathrm{mOsmol} / \mathrm{Kg}$ with purified water and appropriate calibration solutions.

\section{Methods}

Measurements of the osmotic pressure (osmolarity)

The measurement of the osmotic pressure of both dissolution media was performed according to the $4^{\text {th }}$ European Pharmacopoeia (Ph. Eur. 4) monograph, which specifies the freezing point method. The osmotic pressure of each dissolution medium was determined in triplicate.
Table 2: Pharmaceutical preparations under investigation

\begin{tabular}{|llll|}
\hline Drug substance & Drug product & Technol. description & Expiry date \\
\hline \multirow{2}{*}{ Ibuprofen } & IBU-Product A & film coated tablets & 11.2002 \\
\cline { 2 - 4 } & IBU-Product B & sugar coated tablets & 08.2004 \\
\cline { 2 - 4 } & IBU-Product C & capsules & 09.2004 \\
\hline \multirow{2}{*}{ Indometacin } & INDO-Product A & uncoated tablets & 07.2003 \\
\cline { 2 - 4 } & INDO-Product B & capsules & 11.2004 \\
\hline \multirow{2}{*}{ Metronidazole } & METRO-Product A & film coated tablets & 06.2005 \\
\cline { 2 - 4 } & METRO-Product B & uncoated tablets & 09.2004 \\
\hline
\end{tabular}

Table 3: Description of the methods for dissolution testing

\begin{tabular}{|lll|}
\hline Parameter of the method & Method 1 & Method 2 \\
\hline Apparatus & paddle & paddle \\
\hline Dissolution medium & $\begin{array}{l}\text { Simulated Intestinal } \\
\text { Fluid, pH 6.8 (USP 26) }\end{array}$ & $\begin{array}{l}\text { Phosphate Standard } \\
\text { Buffer pH 6.8 (IntPh 3) }\end{array}$ \\
\hline Degassing & yes & yes \\
\hline Volume & $500 \mathrm{~mL}$ & $500 \mathrm{~mL}$ \\
\hline Agitation & $75 \mathrm{rpm}$ & $75 \mathrm{rpm}$ \\
\hline Temperature & $37^{\circ} \mathrm{C}$ & $37^{\circ} \mathrm{C}$ \\
\hline Sampling time & $10,20,30,45,60 \mathrm{~min}$ & $10,20,30,45,60 \mathrm{~min}$ \\
\hline
\end{tabular}

pore size. The dissolution system used was calibrated to meet USP apparatus suitability test requirements.

A UV-Vis spectrophotometer model DU 640i (Beckmann Coulter, Germany) was used to analyze the samples for dissolved drug. The instrument was calibrated periodically according to the Pharmacopeial requirements.

For the $\mathrm{pH}$ measurements of dissolution media an MP $230 \mathrm{pH}$ meter (Mettler Toledo, Germany), calibrated daily, was used.

\section{Buffer capacity}

The buffer capacity was determined according to the titration method and reported as the volume of strong acid added to one liter buffer solution to lower its $\mathrm{pH}$ by one unit. This was determined by adding $1 \mathrm{~N}$ hydrochloric acid solution drop-wise from a $25 \mathrm{~mL}$ buret into the $100 \mathrm{~mL}$ sample buffer solution, with constant stirring, until the $\mathrm{pH}$ decreased by one $\mathrm{pH}$ unit. The investigations were performed in triplicate for each dissolution medium.

\section{In vitro dissolution \\ In vitro dissolution tests were} performed with the paddle apparatus in $500 \mathrm{~mL}$ dissolution media at $75 \mathrm{rpm}$. The dissolutions were run in accordance with the requirements of Ph. Eur. 4/ USP 26. Pre-measured volumes of dissolution media were degassed and then immediately transferred into the vessels. The tablets were placed in the vessels at the beginning of each test and the stopwatch was started simultaneously. Vessels were covered during the run with plastic covers in order to minimize evaporation. The temperature in the vessels was maintained at $37 \pm 0.5^{\circ} \mathrm{C}$ throughout each dissolution run. The revolution rate was checked during all tests. Samples were removed manually using $5 \mathrm{~mL}$ syringes fitted with stainless tubing to ensure reproducibility of the sampling location. Samples were filtered immediately after sampling through $45 \mu \mathrm{m}$ PTFE filter sticks.

The parameters used for the in vitro dissolution testing are described in Table 3. 


\section{Analysis}

The percent of drug dissolved at each sampling time point was determined for each drug product by UV analysis. The UV measurements were performed in all cases at the wavelength of the relative maximum absorption of the drug substance, which was determined prior to each analysis.

\section{Calculation of \% dissolved drug}

Reference standard solutions were prepared by separately weighing portions of the chemical reference standard substance. Reference standard solutions were prepared and processed using the same method as for the preparation of the sample solutions. In order to avoid potential analytical interferences, standard solutions were prepared with aliquots of the same medium used for dissolution testing.

For each individual dissolution media UV-spectra between $200 \mathrm{~nm}$ and $400 \mathrm{~nm}$ of a typical blank solution, a typical sample solution, and the chemical reference standard solution were recorded. The relative maximum of the absorption spectrum was determined. By comparison of the recorded spectra, interference between the drug and the excipients dissolved in the medium was evaluated.

Concentrations of drug were calculated according to the external standard method with a linear calibration plot. The linearity of the calibration function was confirmed for each individual analytical sequence at three concentrations within the working range. The in-study validation for each sequence also included a check of the repeatability by six-fold measurement of reference standard solutions.

\section{Results and Discussion}

\section{Osmotic pressure and buffer capacity}

The results of the investigations on the osmotic pressure and buffer capacity of both dissolution media are given in Table 4.

The two media are practically identical with respect to $\mathrm{pH}$, buffer capacity, ionic strength and osmotic pressure.

Table 4: Osmolarity, ionic strength and buffer capacity of the two buffers

\begin{tabular}{|lccc|}
\hline Medium & $\begin{array}{c}\text { Osmolarity } \\
\text { [mOsmol/kg] }\end{array}$ & $\begin{array}{c}\text { Ionic strength } \\
\text { [mol/L] }\end{array}$ & $\begin{array}{c}\text { Buffer capacity } \\
{[\mathbf{m E q} / \mathbf{L} / \mathbf{p H} \text { unit] }}\end{array}$ \\
\hline $\begin{array}{l}\text { Simulated } \\
\text { Intestinal Fluid, } \\
\text { pH 6.8 (SIFsp); } \\
\text { USP 26 }\end{array}$ & 113 & 0.0720 & $18.4 \pm 0.2$ \\
\hline $\begin{array}{l}\text { Phosphate } \\
\text { Standard Buffer } \\
\text { pH 6.8 (IntPh 3) }\end{array}$ & 115 & 0.0753 & $18.6 \pm 0.1$ \\
\hline
\end{tabular}

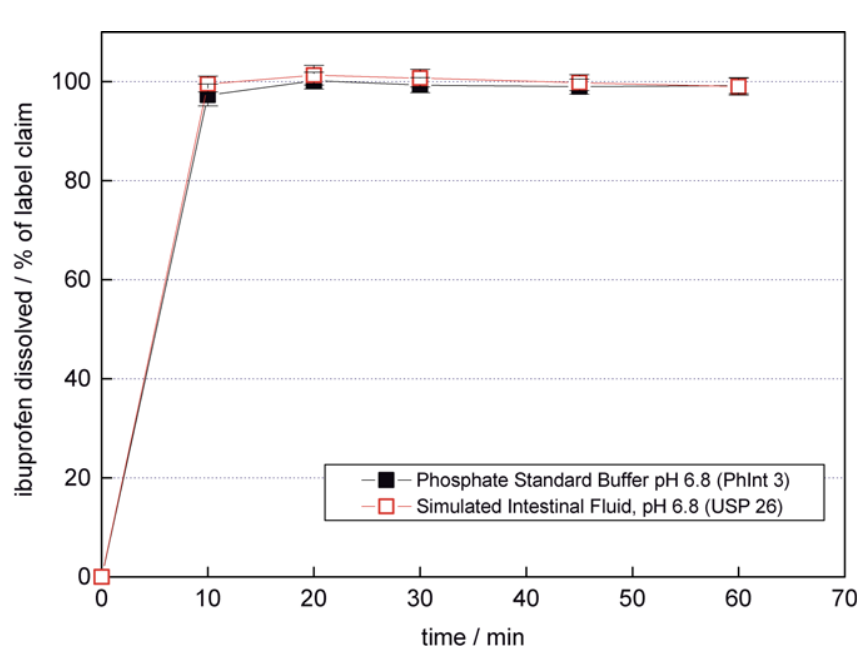

Figure 1.1. In vitro dissolution behavior of IBU-Product A film coated tablets. Arithmetic means $(n=6)$ are shown, standard deviations were within the symbols.

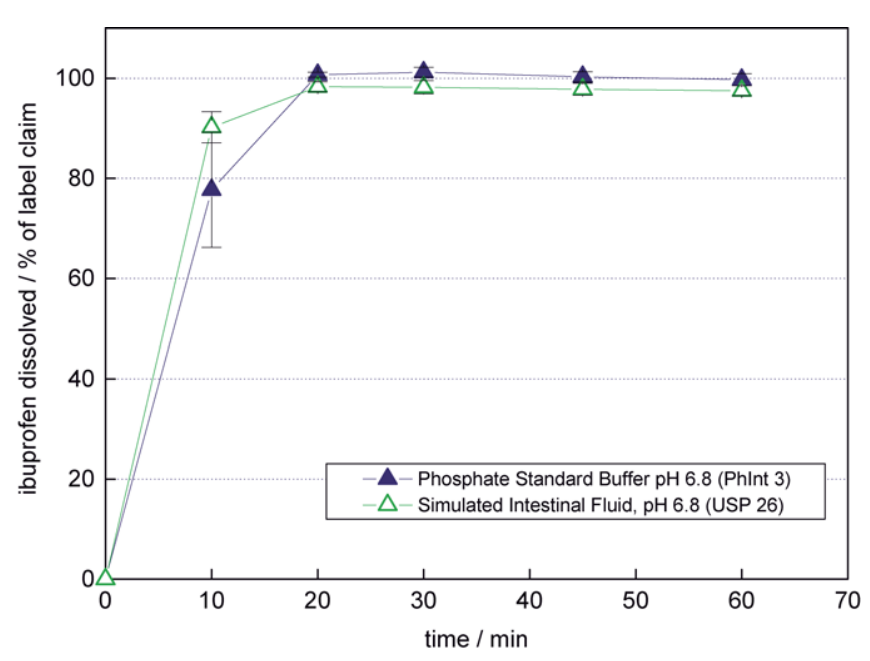

Figure 1.2. In vitro dissolution behavior of IBU-Product B sugar coated tablets. Arithmetic means $(n=6)$ with standard deviations are shown.

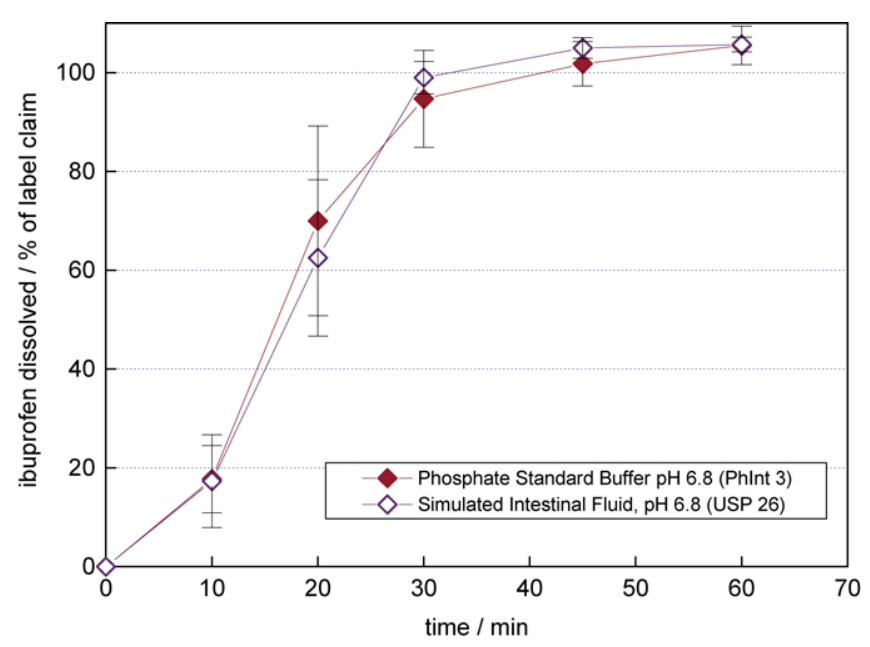

Figure 1.3. In vitro dissolution behavior of IBU-Product C capsules. Arithmetic means $(n=6)$ with standard deviations are shown. 


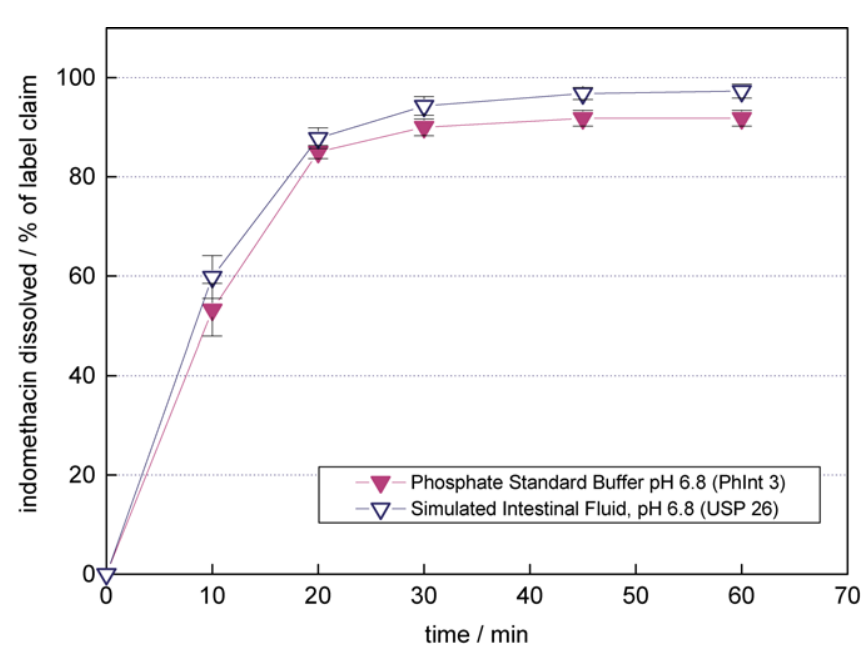

Figure 2.1. In vitro dissolution behavior of INDO-Product $A$ uncoated tablets. Arithmetic means $(n=6)$ with standard deviations are shown.

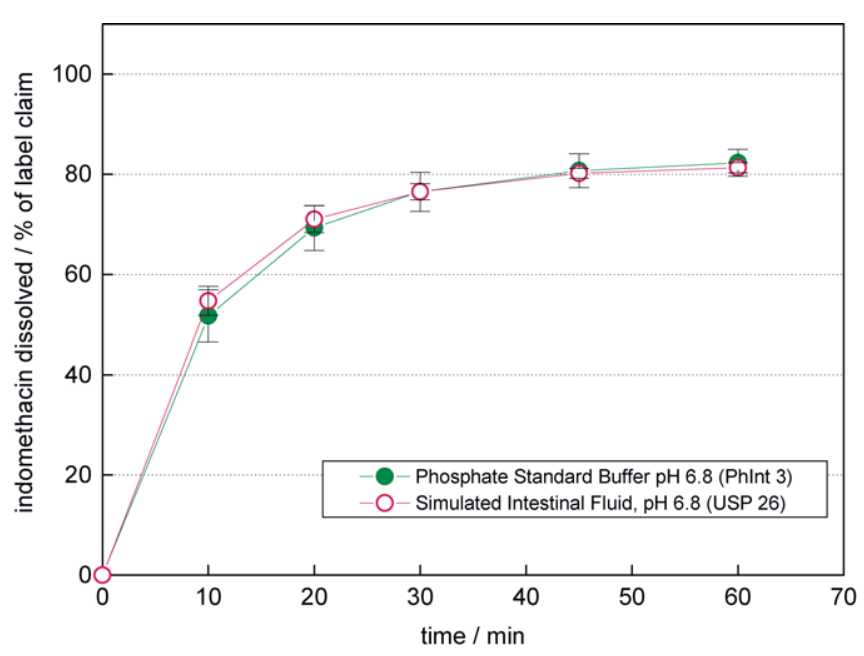

Figure 2.2. In vitro dissolution behavior of INDO-Product B capsules. Arithmetic means $(n=6)$ with standard deviations are shown.

Therefore, dissolution behavior is expected to be similar in the two media for majority of products. Only in a specific case where the cation $\left(\mathrm{Na}^{+} \mathrm{vs} . \mathrm{K}^{+}\right)$may have an influence, would a difference in results be anticipated.

\section{Dissolution Characterization}

\section{Excipient interference:}

Interference of excipients did not exceed 5\% for any of the products tested. Any interference was taken into consideration in calculating \% dissolved, as described in the methods section.

\section{Drug products containing ibuprofen}

Mean in vitro dissolution behavior of various dosage forms containing ibuprofen under the two testing conditions are depicted in Figures 1.1 to 1.3.

No difference in the dissolution behavior of IBU-Product A film coated tablets was observed and dissolution was

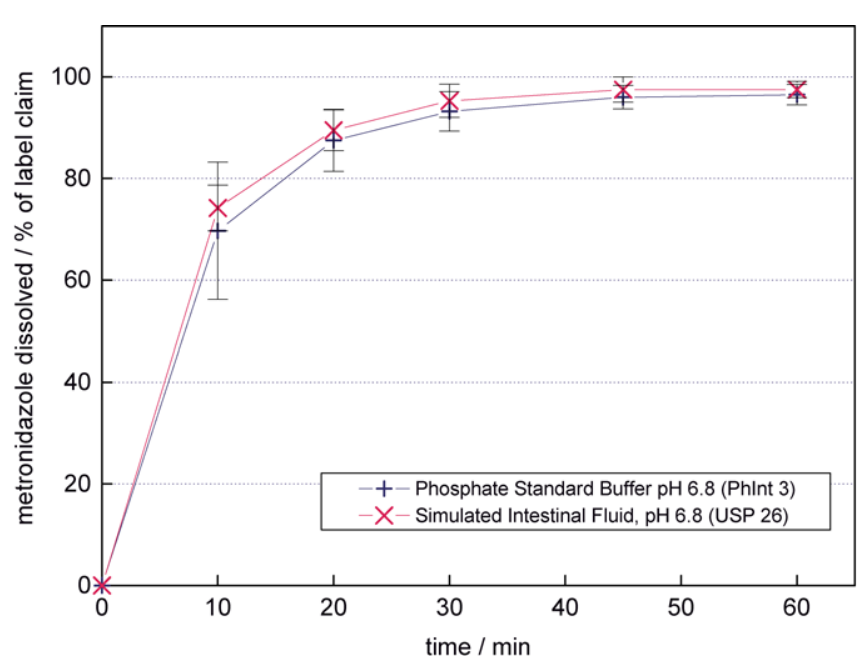

Figure 3.1. In vitro dissolution behavior of METRO-Product A film coated tablets. Arithmetic means $(n=6)$ with standard deviations are shown.

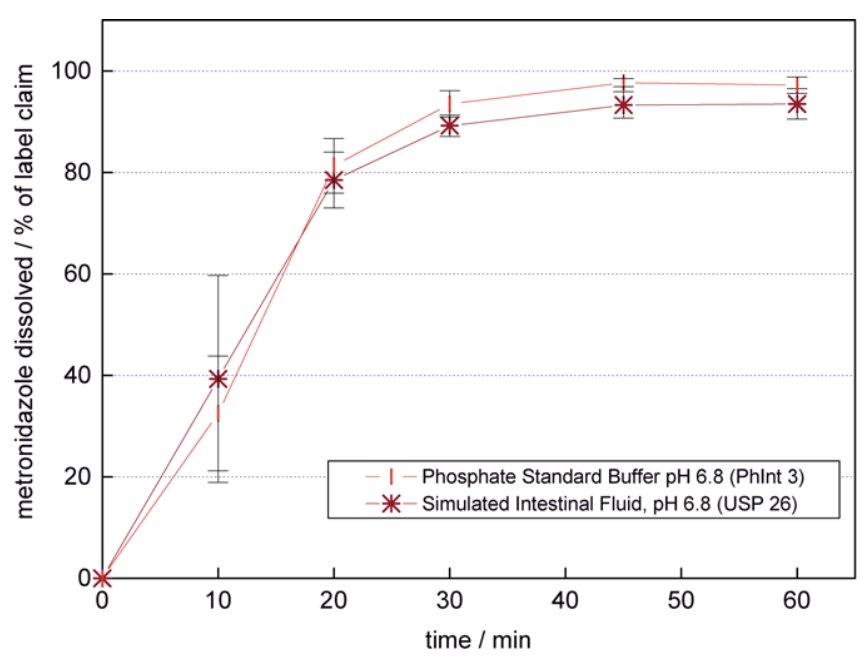

Figure 3.2. In vitro dissolution behavior of METRO-Product $B$ uncoated tablets. Arithmetic means $(n=6)$ with standard deviations are shown

complete in both media within 10 minutes. The dissolution profiles are superimposable and meet USP specifications.

A small difference in the initial dissolution behavior of IBU-Product B sugar coated tablets was observed. In both media the dissolution of IBU-Product B sugar coated tablets was complete within 20 minutes. The dissolution profiles are similar and both meet USP dissolution specifications.

Little or no difference in the dissolution behavior of IBUProduct $C$ capsules was observed. Even though the dissolution profiles for this product are quite different from the other two products, they meet USP specifications. The difference is attributable to the dissolution time of the capsule shell.

Drug products containing indometacin

The in vitro dissolution behavior of two indometacin drug products (one uncoated tablet and one capsule 
formulation) under the two testing conditions are depicted in Figures 2.1 and 2.2.

Little difference in the dissolution behavior of INDOProduct $A$ uncoated tablets was observed. The amount of the drug dissolved in Phosphate Standard Buffer $\mathrm{pH} 6.8$ was slightly lower in comparison to that in SIFsp, but the shape of the dissolution profiles are similar and both profiles meet USP specifications.

No difference in the dissolution behavior of INDOProduct B capsules was observed. The dissolution profiles are superimposable and meet USP specifications.

Drug products containing metronidazole

The in vitro dissolution behavior of two metronidazole drug products (one film coated and one uncoated tablet formulation) under the two testing conditions are depicted in Figures 3.1 and 3.2.

Little difference in the dissolution behavior of METROProduct A film coated tablets in the two media was observed. The shapes of the dissolution profiles are similar and both profiles meet USP specifications.

Slight differences in the dissolution behavior of METROProduct B uncoated tablets were observed. The amount of the drug dissolved at 10 minutes in Phosphate Standard Buffer pH 6.8 (IntPh 3) was slightly lower and after 20 minutes slightly higher in comparison to that in SIFsp. However, both profiles easily meet USP specifications.

\section{Conclusions}

Dissolution results were found to be similar in the three cases investigated. This is not surprising, since $\mathrm{pH}$, buffer capacity, ionic strength and osmolarity of the two buffers are almost identical. In fact, the distinguishing factor between the two media rests solely in the ratio of sodium to potassium. The results of these studies suggest that changing the proportions of these two cations in the medium makes little or no difference to the dissolution results. For the purposes of dissolution testing of conventionally formulated immediate release solid oral dosage forms, the Phosphate Standard Buffer $\mathrm{pH} 6.8$ of the IntPh 3 can be generally considered interchangeable with SIFsp of the USP 26. Only in specific cases where solubility is known to be affected by the cation does one need to be wary about substitution of the two cations for each other. In an upcoming report, we will address this issue using the dissolution of phenytoin sodium capsules in sodium lauryl sulfate containing media as a case example.

\section{References}

1. United States Pharmacopeia and National Formulary, United States Pharmacopeial Convention Inc., Rockville, MD, USA, 25th Edition (2002) and 26th Edition (2003)

2. The International Pharmacopoeia, WHO, Geneva, Switzerland, 3rd Edition, Volume 1 (1979) and Volume 5 (2003)

3. European Pharmacopoeia, European Directorate for the Quality of Medicines of the Council of Europe (EDQM), Strassbourg, France, 4th Edition (2001) 